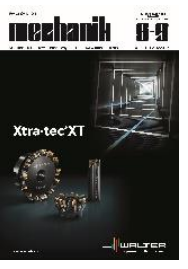

\title{
Development of a mobile robot demonstrator for inspection and engineering works in sewage pipes
}

\author{
JAROSŁAW PANASIUK \\ WOJCIECH KACZMAREK \\ MICHAŁ SIWEK \\ PIOTR PRUSACZYK \\ SZYMON BORYS *
}

\author{
Dr inż. Jarosław Panasiuk (jaroslaw.panasiuk@wat.edu.pl), https://orcid.org/0000-0001-8480-1654 - Wojskowa Akademia Techniczna, \\ Warszawa, Polska \\ Dr inż. Wojciech Kaczmarek (wojciech.kaczmarek@wat.edu.pl), https://orcid.org/0000-0003-3805-9510 - Wojskowa Akademia Tech- \\ niczna, Warszawa, Polska \\ Mgr inż. Michał Siwek (michal.siwek@wat.edu.pl), https://orcid.org/0000-0003-2818-9725 - Wojskowa Akademia Techniczna, War- \\ szawa, Polska \\ Mgr inż. Piotr Prusaczyk (piotr.prusaczyk@wat.edu.pl), https://orcid.org/0000-0002-1347-1202 - Wojskowa Akademia Techniczna, War- \\ szawa, Polska \\ Mgr inż. Szymon Borys (szymon.borys@wat.edu.pl), https://orcid.org/0000-0003-1620-6145 - Wojskowa Akademia Techniczna, War- \\ szawa, Polska
}

Implementation of inspection and engineering works in sewage pipes is a serious logistic and technological challenge. The development of mobile robot construction technology makes it possible to use them in this type of tasks. The paper presents the results of work aimed at developing the construction of an inspection and engineering robot, intended for inspection, repair and installation of tele-technical wires (fiber optic) in sewage pipes with a diameter range of $200 \div 800 \mathrm{~mm}$. The design of the robot as well as the modules of the demonstrator are described (driving module, carrier module, technological module) and the results of tests aimed at verifying the work parameters of the developed demonstrator. KEYWORDS: robotics, automation, mobile robot, sewage pipes, control, fiber optics

\section{Introduction}

Developing a conceptual design and then constructing a mobile robot demonstrator for inspection and engineering works, including works related to the installation of tele-technical cables, is a very complex issue, especially when specific requirements as to the scope, conditions and technology of carrying out these works are imposed $[1,3]$. Designers must know both the environment, in which the robot is to work and the competitive solutions of mobile robots available on the market.

Due to dimensional specificity of the sewage pipes, to which the robot was to be adapted, along with all modules, it was necessary to use very advanced solutions and structural elements.

The demonstrator required the development of a complex software, operating at three levels, i.e. at the level of the control console, chassis module and technology module.

\section{Construction of the inspection and engineering robot}

The design of the inspection and engineering robot demonstrator, enabling operation in sewage pipes with diameters of $200 \div 800 \mathrm{~mm}$, imposed the use of modular construction - both the entire robot and individual modules [1]. Due to the multi-tasking of the developed structure (for inspection and repair and assembly of optical fiber cables), it was decided to divide the structure into the following functional modules:

- drive module;

- carrier module;

- technological module;

- power supply and communication module; 
- software module.

\section{Drive module}

Based on the structural analysis carried out at the stage of construction of the support frame of the mobile module in the initial variant of the developed model, and on the basis of experience gained during the assembly of the frame, it was found necessary to introduce changes in the detailed design and assembly of this module $[2,4]$.

The basic and most important change was the modernization of the support frame. Steel, welded bearing profiles and the multi-part central joint were abandoned. It was found that to ensure maximum rigidity of the structure and repeatability of dimensions, in the case of the production of more robots, it is necessary to eliminate bolted joints and introduce one-piece bearing elements. On the basis of these assumptions, one comprehensive element of the rear housing was developed, which is also a load-bearing element with an integrated joint, and one comprehensive element of the front housing with an integrated joint and with matching sockets for the engine bed. The elements include target mounting holes for housing covers, pneumatic and electrical connectors, matching bearing holes and matching sockets for mounting pneumatic cylinders.

Due to the use of two main housing parts as elements of the chassis supporting frame, additional four elements have been developed to act as covers. Two symmetrical elements mounted from the side are the engine covers, and the other two elements are mounted from the top and cover the back and front of the drive module.

The CAD model of the drive module frame is shown in fig. 1, and in fig. 2 - a view of the robot housing. Silicone gaskets ensuring IP 67 protection class are used between the individual housing components.
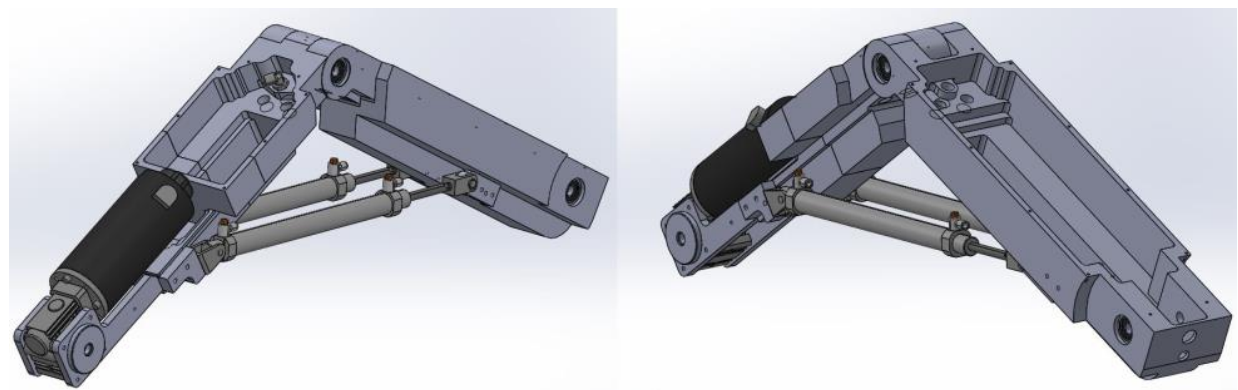

Fig. 1. CAD model of the drive module frame in a broken configuration

a)

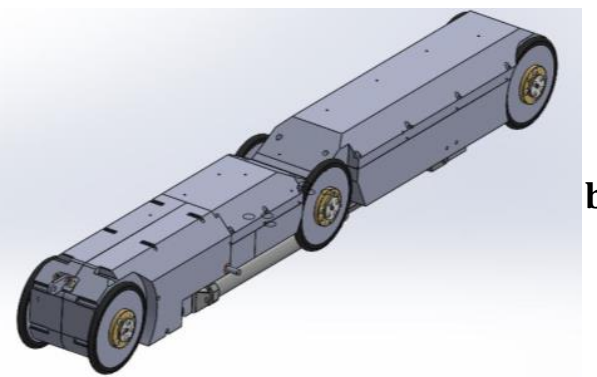

b)

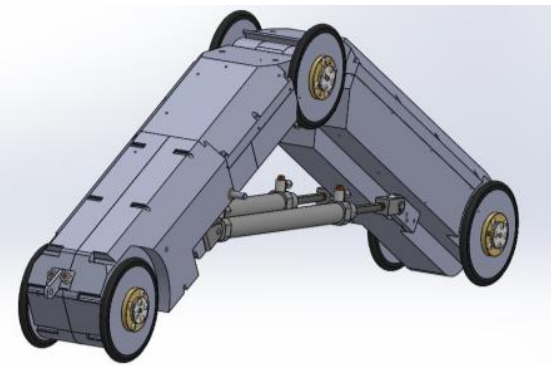

Fig. 2. View of the drive module with housing in basic (a) and broken (b) configuration

\section{Carrier module}

Due to the control, the carrier module is a passive element of the structure. Its main function is to maintain the technological or inspection module in the axis of the sewage pipe [5]. Depending on the diameter of the pipe, in which the robot's work is carried out, the carrier module may be located behind or inside the technological module (in the case of pipes with a diameter of 600 and $800 \mathrm{~mm}$ ).

The use of four wheelsets arranged diagonally (fig. 3) does not interfere with a $25 \mathrm{~mm}$ fiber optic cable located on the top of the pipeline in the engineering version. 


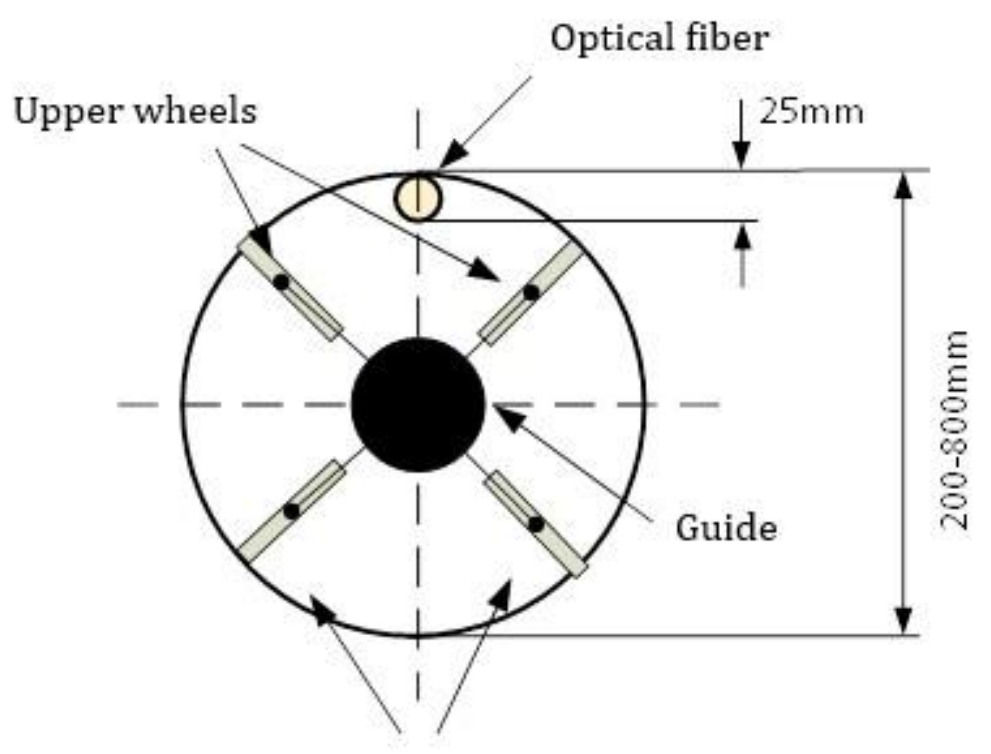

Bottom wheels

Fig. 3. Front view of the diagram of technological modules carrier

\section{Technological module}

In the HDPE tube installation mode, the robot and technological module configuration is different than in the video inspection mode. During installation, the robot moves backwards, pulling along the technological module carrier module with the installed installation module. The installation module is mounted between the mobile robot module and the module carrier module. The fastening tape magazine is intended for transporting 350 $\mathrm{mm}$ of tape supplied by the feeder and cut with a thermal knife integrated with the adhesive feeding nozzle.

\section{Power and communication module}

The developed power supply and communication module ensures:

- reliable transmission of binary and multi-bit control data from the inspection and engineering robot (RII) to the robot control console (KSR) and from the console to the robot;

- automatic signaling of connection establishment (within the meaning of the TCP protocol) and possible loss of communication, regardless of KSR $\rightarrow$ RII and KSR $\rightarrow$ SWR data exchange;

- video signal transmission from RII at a resolution of not less than $1024 \times 768$, from two switched cameras;

- supply of propulsion systems, RII control and technological module;

- communication with the internal RII control system,

- communication between elements of the technological module and controlling their work.

Devices working on board of the technological module require power supply with the following parameters: $230 \mathrm{~V} \mathrm{AC} 50 \mathrm{~Hz}, 24 \mathrm{~V}$ DC, 5 V DC.

Data transmission is carried out using the USB (universal serial bus) protocol, which allowed to reduce the number of wires in the transmission cable between individual robot modules.

\section{Software module}

The on-board operating system of the inspection and engineering robot is equipped with a proprietary application for managing the status data - taken from the Arduino executive modules in the drive module (located in the inspection and engineering robot) and the technological module - as well as control data sent from the CRS.

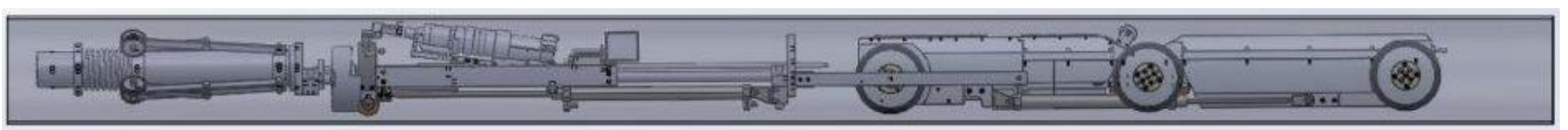

Fig. 4. Robot configuration for a pipe diameter of $200 \mathrm{~mm}$ (gray background is the cross-section of the pipe) - side view 


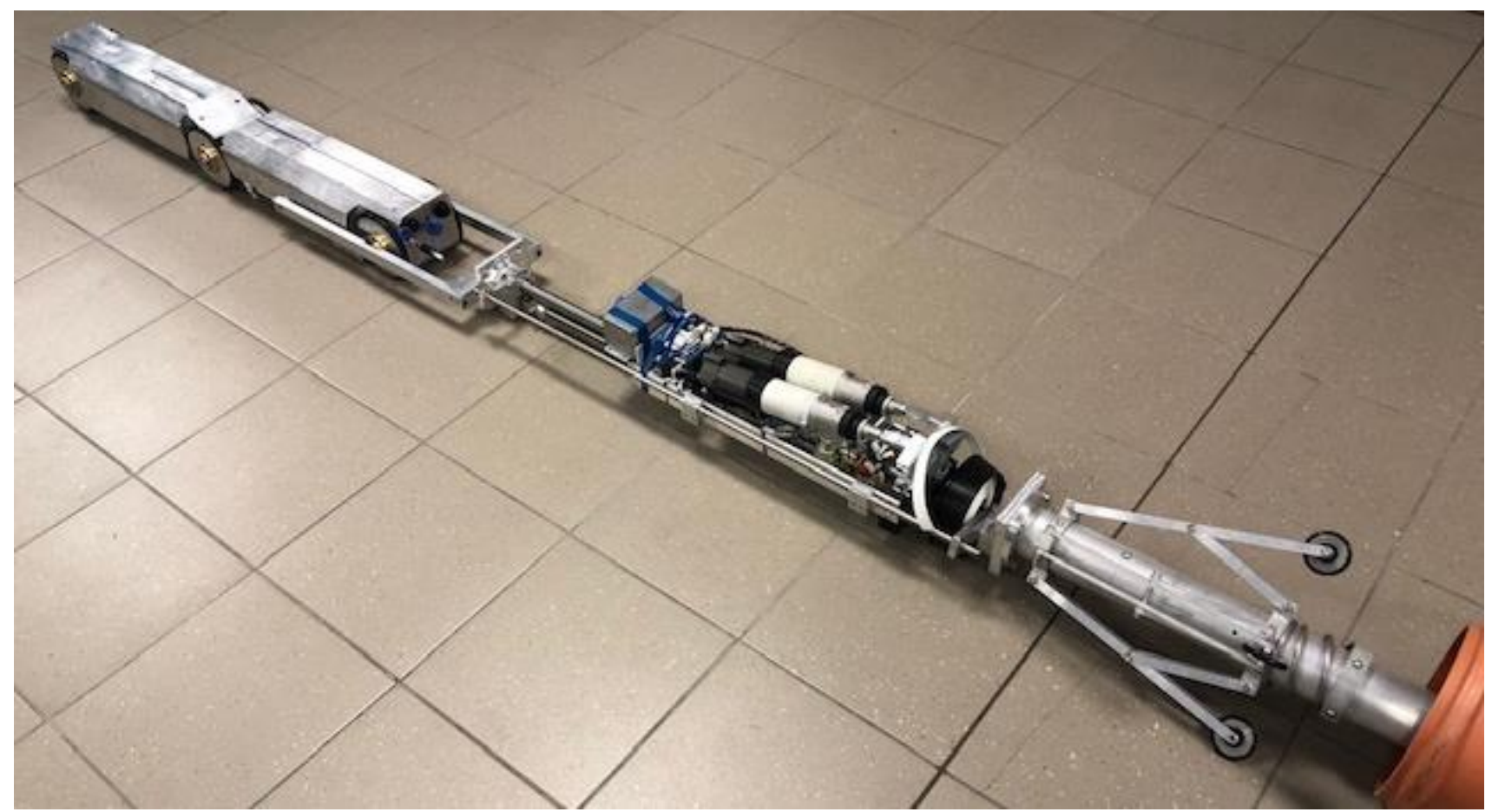

Fig. 5. Configuration of the actual demonstrator for a $200 \mathrm{~mm}$ pipe diameter along with the technological module carrier and the technological module

The application associates data exchanged with the CRS with data transferred between the Arduino executive modules. The control software in the console version acts as an interface, on which the operator carries out the entire robot control process. The operator can both send commands to the robot and observe the image from the camera mounted on the drive or technological module.

\section{Robot configurations for pipes with a diameter of $200 \div 800 \mathrm{~mm}$}

In order to ensure the correct operation of the robot in sewage lines of different diameters, it is necessary to change the configuration of the robot elements - both the driving module, as well as the carrier module or the technological module. Changing the configuration consists in changing the setting and orientation of individual construction modules and replacing the wheels with wheels with a diameter that matches the diameter of the sewage pipe.

Four basic robot configurations are foreseen depending on the diameter of the pipeline:

- for diameters in the range of $200 \div 300 \mathrm{~mm}$ with a main working diameter of $200 \mathrm{~mm}$ (figs. 4 and 5);

- for diameters in the range of $300 \div 450 \mathrm{~mm}$ with a main working diameter of $400 \mathrm{~mm}$ - an element has been added to hold the technological module in a given position relative to the pipe surface;

- for diameters in the range of $450 \div 650 \mathrm{~mm}$ with a main working diameter of $600 \mathrm{~mm}$ - an element has been added to hold the technological module in a given position relative to the pipe surface and the orientation of the carrier has been changed to reduce the length of the structure;

- for diameters in the range of $650 \div 800 \mathrm{~mm}$ with a main working diameter of $800 \mathrm{~mm}$ - an element has been added to hold the technological module in a given position relative to the surface of the pipe and the orientation of the carrier from the previous variant has been retained so as not to lengthen the structure.

\section{Summary}

The developed solution of the inspection and engineering robot, intended for the preparation and laying of tele-technical installations (fiber optic cables), was a complicated process both at the stage of designing the complete device, as well as at the stage of its construction and integration and programming of the control system. Development of the gluing process turned out to be very difficult, especially in the case of pipes with a diameter of $200 \mathrm{~mm}$, in which all robot components, glue and supporting material - tape - had to be accommodated.

Obtained results confirmed the correctness of adopted solutions, and at the same time, provided important hints as to possible structural changes. These changes should first of all consist in reducing the total weight of 
the robot along with individual modules and supplementing the structure with sensory elements that allow for continuous monitoring of the drive parameters of the drive module and technological module.

The paper contains results of work financed from the NCBR funds under the Intelligent Development Operational Program 2014-2020 POIR.01.01.01-00-0893/16 "Development and implementation of a mobile inspection and engineering device (robot) intended in particular for the installation of tele-technical cables (optical fibers) in sewage pipes".

Special thanks go to the representatives of KARTEL S.C. from Warsaw - Jacek Ejchelkraut and Sebastian Brański - principals and originators of the project.

\section{REFERENCES}

[1] Panasiuk J., Siwek M., Kaczmarek W., Borys S., Prusaczyk P. "The concept of using the mobile robot for telemechanical wires installation in pipelines". AIP Conference Proceedings, 29.10.2018 r.

[2] Baranowski L., Panasiuk J. Siwek M. "Use of a Raspberry Pi to built a prototype wireless control system of a mobile robot". Proceedings of the 23th International Conference - Engineering Mechanics (EM 2017).

[3] Iszmir Nazmi Ismail, Adzly Anuar, Khairul Salleh Mohamed Sahari. "Development of in-pipe inspection robot: A review". 2012 IEEE Conference on Sustainable Utilization and Development in Engineering and Technology. [4] Baranowski L., Siwek M. "Use of 3D simulation to design theoretical and real pipe inspection mobile robot model". Acta Mechanica et Automatica. 12, 3 (2018).

[5] Sharanabasappa C. Sajjan, Naveen Srivatsa H.S, Dinesh Kumar P. "Design and development of pipe inspection robot". International Journal of Current Engineering and Scientific Research. 2, 12 (2015). 